Voix et Images

\title{
La Manufacture de machines de L.-P. Hébert : Une machination du texte dans ses effets
}

\section{Ghislain Bourque}

Volume 4, numéro 3, avril 1979

Louis-Philippe Hébert

URI : https://id.erudit.org/iderudit/200169ar

DOI : https://doi.org/10.7202/200169ar

Aller au sommaire du numéro

Éditeur(s)

Les Presses de l'Université du Québec

ISSN

0318-9201 (imprimé)

1705-933X (numérique)

Découvrir la revue

\section{Citer cet article}

Bourque, G. (1979). La Manufacture de machines de L.-P. Hébert : Une machination du texte dans ses effets. Voix et Images, 4(3), 407-435.

https://doi.org/10.7202/200169ar d'utilisation que vous pouvez consulter en ligne.

https://apropos.erudit.org/fr/usagers/politique-dutilisation/ 


\section{La Manufacture de machines de L.-P. Hébert : Une machination du texte dans ses effets}

\section{Les machines célibataires}

Un système de domination quel qu'il soit tombe sous le sens. Le rapport, devenu certain dès l'entreprise de lecture, renvoie la « manufacture " à l'établissement permanent d'une machination. Mais encore, cette domination sous le sens pourrait bien devoir se traduire en une domination du sens, pourvu bien sûr que puissent textuellement se marquer affrontement et opposition. Voilà qui saurait déjà, d'une écriture machinique, en calibrer certains effets.

Mais d'abord, pris dans un procès de référence, et avant tout, puisque aussi bien intertextuel, serait à prélever l'implicite, et parfois même l'explicite région du développement de cette Manufacture ${ }^{1}$. Ici certainement deux champs : I'un où l'explicite, en ce qui a trait à l'œuvre de Raymond Roussel, domine. Père des machines célibataires, il ne manque pas d'être convié en maints endroits du texte. Ainsi apparaît-il d'abord, dans "l'Aqueduc" (le deuxième des quinze récits de la Manufacture) par l'évocation de la "localité de Canterelle" (p. 21) qui comme telle, reprend de façon quasi parfaite le nom du maître d'œuvre, Canterel, de Locus solus. Puis, mis à vif, s'énonce celui moins discret de l'auteur, "Raymond», unique nom dévoilé dans le texte, au cinquième récit ("La Fugue»). (II y aurait encore bien d'autres marques à relever, par citations allusives, où se retrouvent "compas" et "roues à aubes" par exemple, mais vaut mieux céder la place.)

Et où l'implicite, de l'autre, tire du côté de Kafka tout un système dont l'affrontement et le processus, correctionnel, insinuent pour le "village " proposé les conditions d'existence d'une colonie. L'anonymat, à une exception près (Roussel, Raymond en tant que dernier élément de survie), souligne l'écrasement et la compression, non pas humaniste mais textuelle du récit. A savoir : ce qui déjà se pressent comme un travail en réduction de différents éléments. 
Le célibat de la machine

Lieu privilégié de la fabrication, la Manufacture de machines sollicite non seulement l'apparition mais le fonctionnement des machines les plus invraisemblables. Car ici, la manufacture, plus qu'à un résultat, équivaut à un lieu de production; c'est-à-dire, ce lieu où naissent les machines célibataires. Voilà qui prête à la critique.

Il s'agit, devant cet illogisme, de constater de façon machinique d'abord, ce qui constitue le célibat de la machine. Nul intérêt, là, à répertorier l'ensemble; simplement, cependant, un précepte se dégage: "La machi ne célibataire apparaît d'abord comme une machine impossible, inutile, incompréhensible, délirante ${ }^{2}$,"

Et ce précepte dit que pour ce qui a trait à la machine:

a) L'impossible condamne sa fabrication au niveau d'un transfert dans le quotidien;

b) l'inutile dégage de toute raison sociale et mécanique;

c) l'incompréhensible fait défaillir la référence au réel ;

d) le délire laisse libre cours à l'imagination.

Dès lors, tend à se dégager un consensus qui fera pencher l'entreprise du côté de la fiction (celle du plus fort statut pataphysique). Science des solutions imaginaires, la pataphysique, en se moquant du référent, se condamne au célibat.

Ce qui oblige à prendre en compte que chaque machine célibataire est un système composé de deux ensembles, lesquels pour l'instant incitent à penser une sexualité, dont les pôles, répertoriés en mâle et femelle, sont condamnés à ne jamais se rencontrer. Isolés, ils vivent à ne pas pouvoir s'accoupler.

Aussi bien, ce dualisme opère une reconnaissance mécanique. Puisque là des pôles s'attirent en se distanciant. Une coïncidence ou une correspondance à faire jouer en parallèle sexe et mécanique évoque une structure dont ne peut se départir la machine. Cela se rencontrera. II n'y a qu'à pressentir "Robot (I) " (le troisième récit), sorte de prototype textuel, afin d'assurer la vérification. S'agissant de deux boîtes, l'une transparente, l'autre opaque, le constat veut que, d'une part, elles fonctionnent isolément (celle transparente en haut, celle opaque en bas) et que, d'autre part, elles ne se touchent pas.

\section{Le célibat du texte}

Pour ce encore, deux pôles (les mêmes bien que différents) font du texte une explicite machine célibataire. Du moins sacrent-ils tout rituel à célibat. Certains effets obtenus à la fois dans la composition et dans le fonctionnement des robots (au nombre de trois) produits par le texte, relancent en champ scripturel les boîtes, opaque et transparente, déjà advenues. 
Ces effets se répertorient à ras de texte, par le biais de deux inconciliables. Référence et fiction s'arrogent, de part et d'autre, l'entièreté de la double dimension du texte. En ce sens qu'issues des boîtes, elles campent l'idée d'un rapport de forces où se jouent toutes les instances de la domination.

Tel s'expose dans l'organisation scripturelle l'enjeu pris au pouvoir de cette machine textuelle. $A$ savoir que toute intervention prolifère en glissements qui, partant d'opacité $v$. transparence, convoquent d'autres appellations : fictions et référence bien sûr, mais tout aussi bien pratique et théorie. Soit des inconciliables toujours dont le travail nécessite, dans un rapport circonstancié de l'un à l'autre, à la fois mise en domination et prise de pouvoir.

De sorte que, selon un projet plus globalisant, les multiples récits de la Manufacture de machines rendent comptent d'un double effet du texte. Où l'un, effet de fiction, distribue, au fur de l'apparition de machines fantastiques, tout l'invraisembable attaché à certaine fiction de la science, et où l'autre, effet de théorie, élabore au gré du fonctionnement de fantastiques machines, toute la matérialité propre à la science de la fiction.

Que pour dire plus clairement, ici, dans les oscillations allant de fiction à théorie et de théorie à fiction, se rencontre un texte à effet mixte, c'est-à-dire un texte qui élabore une fiction selon son bon fonctionnement théorique et inversement. Texte mythique, puisque aussi bien de l'ordre de l'hermaphrodisme, la Manufacture de machines ne permettra aucune reproduction. Plutôt, investi d'un accouplement permanent, il confortera une sorte de parturition à rebours où toute copulation mènera soit à la réduction, soit à la mort. Le rituel, exhibé à la cohabitation des deux pôles, ne va pas sans risque sacrificiel. Et c'est de ce risque, toujours inscrit dans l'élaboration de la page, qu'en bon célibataire se nourrit toute mixité.

\section{La machine à pôles}

\section{Un texte a-système(s)}

Le premier récit permet la venue immédiate d'une mise au point. $\mathrm{Ce}$ qui adresse, dans les nuances d'un «Discours d'utilité ", l'établissement des règles du jeu. Puisque bel et bien des systèmes sont en cause, il va s'agir d'en délimiter dès l'abord les modes d'opposition.

En manière de commun, deux systèmes, ou plusieurs, s'affrontent à même ce lieu. Ou plusieurs car contre le système s'en amènent plus d'un, inventés de toutes pièces, dans le dessein avoué de subvertir et de renverser l'ordre établi. Voilà qui est campé en clair. 
L'articulation s'annonce selon deux ordres. Premièrement, celui inventé par le système: l'utilité; et, deuxièmement, celui proposé par les inventeurs: l'inutilité. En ce modé d'opposition, pour rencontrer les machines, il faut se faire une raison. Car c'est bien dans la "salle d'attente" de part et d'autre du guichet dit "frontière plus que théorique entre les systèmes suggérés et le système en place» (p. 14) que s'ouvre le jeu.

«Frontière plus que théoriquement» devrait déjà énoncer une organisation de théorie, en ce sens que la frontière à la base serait théorique. La prise de position dès lors s'évalue par rapport au code. Quelques indices déjà :

a) Seules les interventions codées sont acceptées par le Bureau des Brevets;

b) le seul critère de sélection étant l'utilité, on rejette toute invention jugée inutile (par ce simple fait que, non codée, elle risque la mise en cause du système);

c) un mécanisme correctionnel est prévu à cet effet. Une déchiqueteuse et un compresseur démolissent l'invention, tandis que l'inventeur se voit transformé en fonctionnaire;

d) tirée de la boîte à discours, la notion de "message" se révèle être un a-priori à toute invention. C'est elle qui limite et censure dans le sens du code.

Avec évidence, ces données s'emboîtent dans le discours, en vue de conforter certaines valeurs concernant l'utilité. Elles demeurent dans la visée du "discours d'utilité". A noter, par contre, l'envers permis de ce système qui, passant par le truchement d'une boîte, révèle la mise en place d'un miroir et plus directement de son effet. La contre-partie alors se lirait comme suit :

L'utilité du discours: où un parcours inverse stimule le texte. Quelques indices encore:

a) En font partie, en tant qu'objets de rebut, toutes inventions non codées;

b) en tant qu'inutiles, elles s'appuient non pas sur une notion de dressage mais sur la charpente même du discours;

c) à effet contestataire, les inventions non codées proposent deux agressions directes au système: le renversement de l'ordre économique et la subversion du système.

Deux ordres se présentent alors. L'un attaché au code et légiférant à compter d'un a-priori «messagier "; l'autre détaché du code et bien loin de s'inscrire dans les coordonnées du message, renvoyant à l'inutile. Deux mécaniques qui, à propos du discours, semblent en arborer les deux opposés. Chacun, pour la circonstance, y allant tantôt d'un territoire à préserver (le système en place), tantôt d'un territoire à conquérir (les systèmes inventés). 
Étrange manufacture que voilà! L'enjeu théorique, de part et d'autre de sa frontière (le guichet électrifié), rencontre deux pratiques divergentes: l'une relevant du code et faisant figure de reproduction (seules sont acceptées les inventions déjà codées); l'autre hors code ne souffrant aucune altération du déjà en place et confectionnée dans la seule visée de ne pas entériner ou compléter le message. L'un qui limite et récupère, l'autre qui consiste et subvertit. D'un texte, celui-ci tout juste, voilà comment, dès l'abord, fonctionne la mécanique.

\section{Du travail de mine}

Après cette inévitable mise au point, se pose en second lieu le comportement de la machine textuelle.

Avec «l'Aqueduc", le mode d'opposition prend une autre tournure. Non pas qu'il distancie les problèmes de confrontation au code, mais plutôt en ce que cette confrontation, traduite en contestation et subversion, cède l'initiative au «Je».

"Gardien de funérarium», en effet, «je» décide une exploration jusque-là interdite. II s'agit, en l'occurrence, de descendre dans le funérarium, d'y explorer ses galeries, au nombre de cinq mais dont les deuxième et troisième sont inaccessibles, et d'y visiter la cathédrale abritant une «fontaine horizontale» aménagée en véritable «labyrinthe liquide» où l'eau arrive et sort par les mêmes orifices.

Initiative prise par le "Je", cette exploration n'en est pas moins une infraction au code. Maints détails l'attestent :

a) En tant que gardien du funérarium, «Je» a un travail de surface ("bien qu'on m'ait confié un travail de surface") (p. 24) Or, il s'aventure en profondeur: explore le souterrain;

b) la riposte du "système" ne se fait guère attendre. Les “chenillettes foreuses à fraises" travaillent à rétablir l'ordre: "Ces manceuvres [des chenillettes] semblent me rabattre vers l'une de ces oubliettes qui m'éloigneraient de la sortie en me faisant plonger un étage plus bas dans un monde inachevé» (p. 27). Prospection retournée en chasse (et encore chasse contre lui), le «Je" subit le mode de répression du système. Le mécanisme correctionnel déjà enclenché par la "déchiqueteuse» et le “compresseur» se poursuit avec la "chenillette foreuse".

En tout état de cause deux systèmes s'affrontent: l'un activé par le "Je" et travaillant en "surface»; c'est ainsi que l'on peut appprendre que le cimetière sert de «raccourci» aux passants; l'autre assimilé aux chenillettes foreuses et travaillant de façon labyrinthique à creuser toujours plus profondément, car le funérarium n'a pas encore atteint son achèvement. 
Quant au travail de subversion inscrit dans la confrontation des deux ordres (sur et sous), il se traduira à nouveau en termes de message. En effet, le “Je" passant sous alors qu'il n'est habilité qu'à travailler sur, tente, au risque d'être renversé et perforé par les chenillettes, un message souterrain : “avec l'esprit d'organisation que donne le désespoir, j'ai tracé à l'aide d'une tige de métal un quadrillé et, sur ce quadrillé, j'ai placé de mémoire les différents couloirs du labyrinthe" (p. 28). tement :

$\mathrm{Ce}$ qui désormais permettrait une lecture plus nuancée de l'affron-

a) De surface : règne un «Je" dont le rôle est de prendre soin d'un terrain (cimetière) utilisé comme «raccourci» par les passants ; ce «Je» toujours va s'affairer à produire un message;

b) souterrain : règnent des “morts" entretenus par des machines dont le rôle est de creuser toujours plus en profondeur; lieu sans message, le «funérarium», bien au contraire d'un «raccourci», permet la mise en place d'un «labyrinthe».

Voilà qui inciterait à penser qu'un double travail s'effectue dans le texte: l'un de surface, l'autre de profondeur. Celui de surface faisant ceuvre de subversion quand parvenu au souterrain, mais de même contraint, devant la riposte des chenillettes à être relégué aux oubliettes. Ce qui dans le domaine de la subversion laisse imaginer la possibilité souterraine d'une récupération ou d'un renversement.

\section{Portrait-robot d'une opposition}

Passés outre les deux premiers récits, vient à se profiler le portraitrobot d'une première étape. Senti dans l'ordre de la confirmation, le troisième récit, intitulé "Robot (I)", permet une nette schématisation de l'usage machinique qui a présentement cours; ici elle encadre et s'intègre.

D'abord le conflit. Partagé déjà en deux “chapitres», il s'élabore selon trois étapes:

1. Opposition :

a) Celle du “Discours d'utilité", où d'entrée l'inutile s'oppose à l'utile, l'invention à la reproduction;

b) celle de "l'Aqueduc", où la surface s'oppose à la profondeur, le «Je" aux machines.

2. Subversion:

a) Une première où la tentative s'effectue par la création sur le message;

b) une seconde où inversée, se trouve subvertie la création (labyrinthe aquatique) par le message.

3. Correction :

a) Mécanisme opérant d'abord contre l'invention et l'inventeur: il s'agira à grands renforts de "déchiqueteuse" et de "compres- 
seur » de détruire l'œuvre et de récupérer l'inventeur (il deviendra “fonctionnaire");

b) contre le «Je» et le message cette fois, le mécanisme correctionnel prendra corps dans des "chenillettes foreuses" qui, au cours de leur travail, veilleront à reléguer le «Je» aux oubliettes. II s'agit plus ici d'une élimination que d'une récupération.

Faisant bloc, chacun des "chapitres" prend soin de mener aux antipodes. Toujours, président à la mise en texte du conflit deux éléments se contestant. Et ce, tant dans des processus d'opposition que de subversion ou de correction.

Il y a dans l'organisation prescrite une démarche qui toujours plus se récupère en principe binaire. Le premier "Robot" mis en valeur par le troisième “chapitre» permet une nette schématisation de l'usage. Ainsi, peut-on évaluer certaine répartition des pouvoirs en suivant quelques "visiteurs touristiques".

“Cette machine est formée de deux boîtes superposées, l'une transparente, l'autre opaque» (p. 32).

Pas très loin d'une explication littéraire à la Sartre, la machine vient recréer en plus détaillé le fonctionnement jusqu'ici machinique du texte. Cette allusion, transparence $v$. opacité / prose $v$. poésie, pour allégorique qu'elle soit n'en perd pas pour autant son efficacité dans l'organisation textuelle. Le conflit offre en prime l'accès aux territoires conquis par deux types d'écriture. De la sorte, il serait bon, dans un contexte répertorié, d'en établir pour chacun d'entre eux la topologie.

Deux boîtes donc: l'une transparente, l'autre opaque.

\section{Boîte transparente: dessus}

a) Multilatérale et dissymétrique, cette boîte a la consistance du plexiglas;

b) la mécanique ne semble plus exister que pour ellemême (laquelle mécanique est détaillée selon ses rouages propres);

c) boîte pleine d'huile épaisse mais transparente:

d) analogie entre le fonctionnement des rouages et l'activité d'acrobates.

\section{Boîte opaque: dessous}

a) Cube noir aux parois hermétiques, de plastique luisant;

b) cube renfermant - un moteur, censément électrique, mais qu'on ne voit pas; sans bruit ni surchauffe;

c) hermétisme de la boite et donc procédé tout à fait imperceptible;

d) sans aucune analogie. 
Ce qui clairement laisse voir sinon une confrontation ferme, à tout le moins une opposition vérifiable dans l'ordre signalé des contenu et contenant de chacune d'entre ces deux boîtes. En effet, là où l'une expose l'autre masque. II s'agit, intégrée l'opposition entre dessus et dessous, de l'image même d'un équilibre précairement maintenu entre deux tendances scripturelles.

La machine est une productrice de texte. Car dans l'opposition et la superposition de boîtres, où l'une exposée toute entière sous forme de contenu se laisse aisément traduire (analogie avec acrobates), et où l'autre toute entière contenant ne souffre de par son hermétisme aucune analogie, se laissent déchiffrer les coordonnées à la fois du mot et du texte, adressant simultanément à l'opacité et à la transparence le littéral et le référent. Pas tant que cette adresse cependant ne condamne tout le jeu du texte. Se vérifiera, par ailleurs, avec quelle souplesse finira par s'organiser cette opposition. Le littéral d'ici ne sera pas le littéral de là-bas. Son opposition d'ailleurs au référent l'amènera vers des penchants fictionnels. En ce sens qu'opposé au référent, il s'établira autant pour marquer la matérialité de l'écriture que pour souligner son mode de production.

Mis en suspension, les deux systèmes s'opposent et, ce, sans se toucher. ("La boîte du dessus semble en suspension, celle du dessous lui fournissant appui mais à distance par un procédé absolument imperceptible. Le fabricant lui-même se refuse à donner plus d'explications sur le contenu de la boîte noire" [p. 34].) A les considérer selon certaine relation de pouvoir, une manière d'égalité (au sens ou aucune ne semble véritablement avoir autorité sur l'autre) domine. Les deux boîtes, de par leur cohabitation textuelle, exhibent l'articulation de deux systèmes, opposés mais ne s'affrontant pas. Cela ne s'entendant que dans l'évaluation d'un pas encore.

\section{Une inversion dans le pouvoir}

Composée de cinq récits, la deuxième série suivie fait à nouveau bloc. Il s'agit en l'occurrence, parcourant une trajectoire assez similaire à celle des trois premiers, des récits quatre, cinq, six, sept et huit qui, pris dans l'ordre, tracent de nouveaux contours à l'équilibrage du littéral et du référent. La limite posée à compter du second Robot ( «l'Extracteur de jus"), à elle seule et de façon schématique, marque la similarité avec le premier bloc. Portrait-robot à nouveau, cette relance affuble une nouvelle étape de la machination.

Mais une étape distincte et différente, puisque bien au-delà de l'analogie. Laissant d'ailleurs, pour ce qui a trait au premier bloc (récits un, deux et trois) la subversion dans le détail d'une machine établissant en clair le mode d'opposition (transparence / opacité; référence / litté- 
ralité), une nette démarcation s'instaure à compter de l'opérativité même de ce mode. Ce qui devient, passée l'opposition et le déséquilibre aidant, une manière de mise en inversion du texte. A laquelle les points d'ancrage demeureront en champ référentiel puisque le texte, pour l'efficacité de son déséquilibre, devra, sur la lecture produite, piéger par le sens.

Travail du référent et travail du sens, on assistera tantôt à l'effacement de l'un dans l'autre, tantôt à celui de l'autre dans l'un. Où l'un, le référent, sera pris en. tant que transparence et où l'autre déterminera une possible direction à cette transparence. Ainsi se présenteront de récit en récit selon les diverses étapes de la machination, de possibles référent sans sens et sens sans référent. Allons-y voir.

Le détail pris en lui-même oblige à une avancée récit par. récit. Aussi l'entrée du déséquilibre devra se marquer dès “les Cogneurs" (premier récit de la deuxième série).

\section{Référence défaillante}

Pour qui veut lire à même la référence, implicite ou explicite, une exposition' à la défaillance prévaut. En effet, le terrain n'offre aucune sécurité. Mouvant, le sens, établi au fur et à mesure des indications topologiques, ne permet qu'une approche déroutante dont l'élaboration conteste en permanence l'unité du texte.

Bien sentie, cette défaillance travaille à mettre en péril la stabilité de la référence. L'effet, dès lors, d'un déséquilibre impose aux "Cogneurs" une activation en position de piègé. Il suffit de la mise en texte du comportement des dits "Cogneurs", d'en prélever quelques marquants indices:

a) Instabilité dans la référence et dans l'identité : les “Cogneurs" s'énoncent en tant que "résidus de peuples nomades", donc sans réferent fixe et par surcroît difficiles à joindre. D'ailleurs ne frappent-ils pas aux portes sans même se présenter...

b) Seconde caractéristique: ils se déplacent en fonction des vents. Ce qui, par l'instabilité du référent, les incite et les oblige à aller dans tous les sens sans pour autant qu'il y en ait un de privilégié. Signalons, de plus, qu'ils ne se manifestent qu'à l'obscurité, ce qui, de lien en lien, ne permet pas la transparence.

c) Quant à leur quête, elle pourrait s'établir au niveau d'un simple point de référence. Nomades se heurtant aux sédentaires, ils attestent un certain déséquilibre. "lls cherchent, c'est l'évidence même, mais ce qu'ils cherchent ils ne pourront le découvrir chez nous, et même s'ils le découvraient, ils ne pourraient pas l'emporter avec eux (p. 40). En ce sens qu'on n'emporte pas avec soi le référent mais qu'on s'y attache et s'y fixe. 
d) Les “Cogneurs" ont pour fonction d'abuser les gens. Cognant aux portes, ils incitent les sédentaires à ouvrir pour rien : «Quand ils ouvrent, ils sont toujours désappointés de s'être laissé encore une fois abuser» (p. 40). Rien d.autre pour lors, ne tend à cette ouverture une référence prise comme piège.

Pas moins loin que là, dans cette ouverture pour rien, la défaillance se fait sentir. Ici le référent est nomade. Voyageant dans tous les sens il abuse par sa non-fixité. Il cogne mais ne se présente jamais. La porte cependant reste ouverte : à tous les sens sans référent fixe.

\section{Référence inexacte}

Lieu d'un autre déséquilibre, l'opposition se traduit, dans l'étalage de deux concepts (théorie/pratique), en un affrontement. Intitulé "la Fugue", le cinquième récit rend compte d'un équilibre brisé dans la configuration du référent.

Le débat ici s'est quelque peu décalé. Considérant quelque pernicieux rapport (théorie $v$. pratique), il s'agit en l'occurrence de vérifier la concordance d'un nouvel équilibre. Pour ce faire, arrive au texte une démarche, celle de Raymond, planifiant en manière de fugue une visée strictement définie, soit: vérifier si la pratique est comme la théorie à savoir être à même de constater si les images vécues sont comme les images apprises dans les livres.

Parlant dans un texte de la possibilité de vérification d'une théorie par la pratique, penche déjà du côté de la théorie le lieu privilégié du référent. En ce sens que l'expérience tentée s'articule dans le cadre d'une pratique d'écriture devant ou non être en équilibre avec son référent. Cette pratique, campée dans un mode de comparaison avec le référent, invite dès lors à regarder du côté de la fiction.

II s'agirait donc d'opposer référent et fiction comme on oppose théorie et pratique; l'ouverture se jouant toujours dans la vérification de leur propre équilibre.

En ce qui a trait à la démarche de «Raymond ", l'hypothèse de départ sera de vérifier si la "locomotive" étudiée dans les livres est identique à celle de la pratique. Aussi se rend-il à la gare :

"La locomotive diésel dont il avait étudié les dessins au point d'être sûr de la reconnaître malgré les perfectionnements techniques qu'on aurait pu lui ajouter depuis la publication de l'encyclopédie, la locomotive avait été remplacée par un engin triangulaire formé de deux bras articulés retenus au sommet par d'épais cercles de fer tournant autour d'un moyeu... (p. 46). Ce qui, selon ce constat, confirme en clair que la pratique se révèle toute différente de la théorie; ou encore, que la fiction est différente du référent. La locomotive apprise dans un texte est différente de la locomotive produite par un texte. Bien loin d'un équilibre permis entre théorie 
et pratique, ou référent et fiction, c'est à l'inexactitude et au leurre que conduit la confrontation.

De ce leurre, maintenant, reste à dégager la trajectoire du sens qui, dans l'inexactitude du référent ou de sa non-concordance avec la fiction, invite à la fugue.

Pour cause, ici, le sens attribué à la locomotive prend valeur de "voyages", mais à cette retenue près que des voyages appréhendés se produit une trajectoire piégée. D'abord quelques éléments :

a) Tout incite à penser qu'on voyage sur place. Lieu de départ et d'arrivée, la gare canalise l'entier déplacement. Ici, point de mobilité. Le voyage s'effectue dans la seule gare. Aussi tout y est factice :

- billet hasardeux et erratique et de plus sans itinéraire aucun; - le voyage se fait sans embarquement puisque la locomotive ne tire pas de chariot;

- les billets sont remboursés sans problème dans la salle d'attente.

b) Ce qui, en comparaison avec le récit précédent, argumente à l'encontre d'un sens nomade. La fugue, effectuée en domaine référentiel (la locomotive pratique est bien loin de la locomotive théorique), tire vers des voyages qui n'ont pas de sens. Où plutôt que d'aller dans tous les sens, comme c'est le cas des cogneurs, l'invitation permet au sédentaire le voyage sur place.

c) A ce récit, vu d'abord l'inexactitude de la référence, l'inversion dans le fonctionnement piège par déséquilibre. La fugue pour certains se révèle fatale : "ll y a des voyageurs très obstinés que l'aspect grotesque de la machine n'a pas découragés et qui se sont installés du mieux qu'ils pouvaient en s'accrochant à l'un des bras. On ne les a pas revus" (p. 49). Partant d'un référent inexact pour des voyages qui n'ont pas de sens, ils subissent le sort réservé à ceux qui s'aventurent dans un système de consommation sur place. Dévoreuse de voyageurs, la locomotive utilise la gare pour fins de fausse représentation.

\section{Référence incomplète}

Ici, loin d'une simple comparaison entre théorie et pratique, c'est d'un travail de production que s'approche le texte. Rapport de l'objet sur plan à celui créé, ce prochain récit propose, dans les coordonnées d'un trapèze, un déséquilibre qui transforme (voir le sixième récit, "Lettre à propos d'un trapèze»).

En l'occurrence il s'agit d'un «plan" à mettre en «pratique». Où, à nouveau, le «référent» se révèle insuffisant, compte tenu d'abord du manque d'habitude pour les producteurs à construire l'objet d'après les plans, et ensuite de ce qué ledit plan se révèle incomplet. 
Proposé en ces termes, le déséquilibre à nouveau se greffe à un mécanisme d'inversion. C'est d'ailleurs ce qui en constitue le piège.

La situation du problème se pose telle que, s'agissant de la construction d'un trapèze, il se révèle :

a) Inhábituel de construire l'objet d'après les plans;

b) que d'habitude on fait le plan d'après l'objet créé;

c) que, par conséquent, pour ce qui a trait à la production en cours, "l'écart entre le plan et les matériaux susceptibles de servir à sa réalisation était trop grand. Entre ce que le regard considère sur le papier et ce que les mains assemblent, il y a un gouffre insondable» (p. 54).

Ce qui conforte l'inversion dans ce qu'elle a de plus pertinent sur le plan de l'écriture. Car, loin de la théorie, loin du référent. Et, en ce cas, ce que la pratique propose c'est de sans cesse vérifier une incomplétion. Si bien que non seulement le fait de travailler à compter du référent se révèle incomplet, mais cela constitue également un piège : un homme n'est-il pas déjà mort en tentant de travailler sur l'appareil... .

Le déséquilibre ressemble ici à un fossé. Le référent ne peut sans risques permettre (on pourrait tout aussi bien dire soumettre) la pratique.

Ainsi, les avatars d'un équilibre impossible aidant, un pernicieux retournement fait passer le pouvoir dans le camp de la pratique. Au fur et à mesure du travail, voire même de son abandon, une transformation s'opère. Le point d'arrivée mystifie le sens de celui de départ. Conçu à la manière d'un «trapèze " le plan, une fois soumis à la pratique, tourne à l' "épouvantail". À savoir qu'en sens inverse ce qui auparavant permettait l'envol désormais le chasse.

La mystification dès lors se révèle de taille: l'incomplétion du référent appelle la transformation du sens, voire même, dans le domaine référentiel, une feinte. Déjà commencent à se faire sentir les effets inévitables de tout mécanisme de production.

\section{Référence piégée}

Activée en nocturne, la démarche du "passant" dans "l'Activité cérébrale des gardiens de nuit " (septième récit) commande un travail non voilé sur la référence. Le piège fonctionne plus ouvertement encore en attirant d'un point de vue théorique une pratique qui, dans le sillage de la feinte, supplée au transfert. Autre qu'une métamorphose, le sens incite la référence à un déplacement et à un remplacement.

Cette mise en déséquilibre instaure chez le passant une démarche qui, suivie, montre les diverses étapes du piège :

a) Présentation du "crâne de bœuf à moteur". Défini à l'abordage en tant qu'objet sans utilité, il fait pencher l'interprétation vers ce 
qui n'a pas de sens. Machine des plus curieuses, inventée pour la seule distraction, elle offre au passant sa gratuité.

b) Mais la gratuité n'est qu'un piège à théoriser. L'incitation opérée chez le passant oblige à certaines hypothèses tant sur la nature que sur le fonctionnement de l'objet. Aussi, le travail du passant consistera-t-il à tenter d'accoler un sens à la machine. Et c'est dans cette opération que s'évaluera le piège. Tombant dans l'ordre des hypothèses, le passant y perd peu à peu son propre référent.

c) L'attraction cependant se joue dans un fonctionnement inversé : «Ces crânes présentent une autre particularité : arrivés au terme de leur course, ils émettent un sifflement, un sifflement lointain qui s'accentue un peu à la manière du sifflement des obus. II n'est pas suivi d'explosion. L'explosion précède, elle a lieu au ralenti : c'est le déplacement" (p. 63). L'explosion précède et c'est elle qui attire. La venue du passant n'étonne guère puisque, comme le lecteur, il se trouve pris dans un système à inversion. Ce qui cependant impose à la théorie son piège: “c'est le déplacement".

d) Changement de sens et déplacement de la référence, s'esquisse en ces termes le transfert. S'emparant du crâne le passant subitement se trouve investi par ce dernier; l'échange se faisant du cerveau du passant à celui du crâne trouvé: «Survient un échange, le gardien de nuit ne peut être perdant; finies les longues veilles, fini ce travail absurde sur des mécaniques fragiles: il peut enfin rentrer chez lui, plonger dans un lit qu'il reconnaît pour l'avoir maintes fois emprunté dans un autre corps, et dormir les deux mains sur la tête sans risquer de se piquer à des cornes pointues. Pour le passant, c'est le début d'une nouvelle existence, noctambule, à faire circuler sa conscience à ras de sol dans des voiturettes qu'il ne faut pas perdre de vue" (p. 65). Nul doute que pour avoir cédé sa référence, le passant prend un autre sens: Ici, comme toujours, à inversion le transfert confirme le piège. Condamné «à faire circuler sa conscience à ras de sol » (p. 65), le passant désormais vaque à une activité nocturne. Ainsi donc la référence est piégée. Et de plus, elle opère contre celui-là même qui l'arbore. Appelée au transfert, elle démontre une autre virtualité : soit celle de ne pas avoir de sens fixe.

\section{Référence extraite}

En manière de clôture, une nouvelle fois le robot fait loi. (Nous en sommes au huitième récit, «l'Extracteur de jus (Robot II)».) Campé tout comme ce fut déjà le cas au troisième récit, en bout d'étape, il schématise et radicalise une trajectoire. Son travail, en tant que second robot, porte, dans le détail d'une remise au point d'éléments machinaux, une ultime secousse à la référence. 


\section{a) L'affrontement}

Avant l'aveu d'un déséquilibre, il va de soi qu'à nouveau s'exposent les pôles devant permettre contestation et affrontement. Déjà pressentis cependant, ils n'incitent guère à la surprise. Tout au plus nuancent-ils une mise au point antécédante, dont l'articulation principale était légitimée par l'opposition de deux boîtes: l'une opaque, l'autre transparente. Ici se rencontrent et s'affrontent les tenants de ces deux positions :

1) Rangée du côté de la boîte opaque se retrouve «la phrase rythmée et entraînante d'un moteur électrique» (p. 69). Lequel moteur nous est donné comme se situant "sous lui", et comme entraînant toujours un peu plus de «lui " vers la noirceur.

2) Du côté de la boîte transparente, et comme obligatoirement devant se situer au-dessus, se rencontre un «Je» dont la localisation rend inévitable l'affrontement. Ici opposée à la noirceur matérialisée par le bas, se détache le détail d'un autre habitat :

«Ce qui le frappa, dès l'abord, ce fut l'extrême propreté de la pièce, la transparence sans tache de la vitre, les planchers, le plafond et les murs recouverts d'une céramique blanche" (p. 71).

Donc d'emblée démarquées, et ce toujours selon les éléments permis de la robotisation, opacité et transparence partagent les frais d'une machination. Se rencontrant sans pour autant se toucher, ils arborent un indissociable processus où, à tout moment, se jouera la subsistance de la référence: "ll se découvrait à chaque rupture, désemparé comme si entre son existence et celle du moteur électrique s'exerçait un rapport étroit, un lien de nécessité " (p. 69).

\section{b) Le déséquilibre}

Ainsi d'ailleurs se pointe le processus d'extraction du référent. Dans la mise en opposition, il s'agira de replacer, par rapport à l'enjeu, chaque élément dans son camp. Du moteur au "Je" doivent d'office se pointer les deux tendances du texte:

1. Moteur à la "phrase rythmée et entraînante" pour la matière fictive;

2. «Je» baignant dans la transparence pour la matière référentielle.

Le travail se faisant ici déterminera la mise en déséquilibre de ces deux pôles selon l'extraction de l'une par l'autre. Ce qui, dans le simple énoncé du titre «l'Extracteur de jus», pose au “Je» des problèmes d'approvisionnement car, problèmes de jus, problèmes de je, l'extraction dès lors, par simple jeu du moteur, vient s'effectuer contre la référence. Tirant le jus, c'est à l'annulation du «Je» (de son jus) qu'elle appelle. 
Divers éléments distribués de façon éparse rendent un compte précis de ce déséquilibre. D'abord le transfert intermittent de la transparence à la noirceur témoigne d'un glissement à l'endroit du “Je». Puis, par contamination, les pertes dans le comportement de ce même "Je" réalisent l'extraction de la référence. Ces pertes se situent surtout au niveau de la mémoire. En effet, ce «Je»n'a plus de passé, voire même plus d'identité :

“ll devait donc procéder autrement: en premier lieu, s'identifier... » (p. 70). Ce que, sans succès, il tentera de faire puisque tout au plus réalisera-t-il son état de robot. II y a donc là la conséquence d'une rupture totale entre le "Je " et le référent, à telle enseigne que les seules sensations permises se rattachent à la dépendance et à l'impuissance. "Mais pour cela, il avait besoin du moteur, d'une aide extérieure sur laquelle il n'exerçait aucun pouvoir" (p. 73). "ll avait donc besoin d'un moteur, comme ces grands malades qu'il avait déjà vu reliés à des poumons artificiels. Sauf qu'il existait encore une marge entre ces patients et le mécanisme qui les maintenait en vie. Alors que pour lui, depuis l'intervention des cliniciens le moteur constituait une partie intégrante de son organisme» (p. 74). Chez «lui » nul doute que l'abolition de la marge invite à l'envahissement par le moteur. À ce titre faut-il, de marge abolie, prêter extension jusqu'aux abolitions suivies de la mémoire du «Je», et du référent. "Partie intégrante", le moteur ne permet plus de distance par rapport au texte, de référence par rapport au «Je».

\section{c) L'inversion}

Problème de dépendance et de nécessité, ce lien entretenu du “Je" au "moteur" affiche plus de déséquilibre. Contaminant tout le comportement de la transparence il officie de cœur, organe avec lequel il n'est pas sans rapport analogique: "Sans pouvoir établir d'échelle de comparaison sûre, il finit par se dire qu'il ne devait pas être plus gros que le poing... Qu'il ne présentait aucune des qualités élaborées auxquelles il s'attendait. "Un moteur d'aspirateur, non plus petit encore et moins puissant, un moteur de réfrigérateur» (p. 72). Le renversement de la transparence vers la noirceur campe son lien d'opération dans un phénomène d'inversion. En effet, si l'analogie entre moteur et cœur (le cœur étant ici comme le moteur: de la grosseur d'un poing) se trouve confortée, ce n'est pas sans accroc dans leur mode de fonctionnement. À savoir que, plutôt que de propulser et de réchauffer comme tout cœur digne de ce nom, le moteur selon deux comparaisons légitimes, aspire et réfrigère. Ce qui, outre le fait d'inverser le mode d'activation, permet l'extraction la plus complète.

II y aurait donc, à compter du moteur, la confirmation d'un dispositif piégé mis en position dominante depuis le début de cette seconde partie. Ce dispositif, établi de manière à provoquer le déséquilibre, joue, depuis le quatrième récit, à inverser et à renverser le référent:

1. Quatrième récit: Les cogneurs sont désorientés. Ils ont perdu leur sens. 
2. Cinquième récit : Raymond voyage dans une gare où une lócomotive n'a ni wagons ni voyageurs, pour n'aller dans aucune direction.

3. Sixième récit : Un trapèze se transforme en épouvantail.

4. Septième récit : Un passant échange son référent avec un gardien de nuit.

5. Huitième récit: Un moteur mis en remplacement du cœur travaille à extraire toute référence.

A toutes fins pratiques, au fur et à mesure des récits, finit par s'énoncer un nouvel état du texte. Ce second robot, plus que de figurer le mode d'opposition présent dans tout texte, soit le rapport littéral/référentiel, offre le résultat précis d'un affrontement de tous les instants. Perturbé de toutes parts, le référent s'affaiblit puis, renversé, cède le pouvoir. Non fiable, il abdique à rendre ou à donner du sens à la fiction.

\section{Les procédures correctionnelles}

Une fois pour toutes la lecture est piégée. Sa pratique, inéluctablement sentie comme un rituel machiné de longue page, mène au sacrifice. Servant d'appât au fur et à mesure des récits précédents, le déséquilibre entraîne vers un rituel sacrificatoire. Si bien que désormais lire aboutit à une condamnation, laquelle, selon toujours l'idée d'un rituel, conforte, au détriment de l'autre, la concentration d'un camp. En effet, le sacrifice du référent porte en référence le sacrifice. Cela saurait articuler en clair le résultat du déséquilibre. A savoir que désormais le pouvoir d'une boîte sur l'autre semble acquis, et que cette acquisition permet l'organisation d'un certain hermétisme. Ici on a affaire à un système clos. Les machines exposées ne font plus le procès de la référence et de son sens, soit au profit d'un déséquilibre, soit au profit d'une prise de pouvoir.

Dès lors, plutôt qu'à un affrontement à l'exposition des deux pôles (théorie/pratique référent/fiction), on assiste au maintien concerté de ces mêmes pôles en vue du fonctionnement autosuffisant de chacune des nouvelles machines mises en cause.

Loin de créer un déséquilibre ou de manifester une prise de pouvoir, la confrontation des pôles s'effectue sur la base d'une complicité. Complicité qui, au préalable, a su ramener en champ fictif l'entièreté du domaine référentiel. Car loin de la théorie, loin de la référence : ici la fiction s'affaire à récupérer tout travail du sens. En ce que d'abord elle se produit elle-même, et qu'ensuite, dans sa production, elle exhibe deux pôles. Cela faisant que, là où plus haut elle provoquait des ratées entre théorie et pratique, par exemple, s'instaure une démarche offrant au texte une ou des machines autosuffisantes. 
Il faut donc parler, en vue d'une explicite planification, d'un travail de récupération en ce que le sacrifice du référent sera corrigé par une référence au sacrifice, puis d'un mécanisme de correction, où semble permise une extension punitive à la récupération, cela d'ailleurs ayant ses aboutissants dans la "cage libératrice".

L'inversion déjà active au cours des cinq récits précédents, œuvre de pair avec un mécanisme de correction. A savoir qu'ici, invitant à quelque renversement de type involutif, elle punit. Au service de la concentration, elle convie toute lecture à réaliser encore une fois de stratégiques glissements.

\section{Envoutement par la langue}

Les deux pôles entendus de cette nouvelle fiction œuvrent à faire osciller le texte entre la récupération et la correction. S'agissant des émanations de sept récits qui restent, il faut voir comment de l'une à l'autre, le texte promène ses effets.

\section{a) Récupération à temps}

Invitant d'abord la lecture à prendre connaissancè de són organisation temporelle, le récit dit "l'Horloge musicale" campe on ne peut mieux les deux extrémités de la "langue» : "L'action conjuguée du bloc Quatre et du bloc Cinq constitue un système d'horlogerie" (p. 80). A savoir qu'ici s'organise un système temporel formé de deux "Blocs" à la fois distincts et complémentaires, où l'un, le Quatre, offre, de par un mécanisme formant un double $z(Z)$, un parcours de la langue stylisant le sablier; et où l'autre, le Cinq, en forme de plateau tournant, joue le rôle de mécanisme régulateur.

Ce qui avec évidence ramène la présence des deux boîtes orginelles: l'une transparente, et donc convoquant la référence au sablier; l'autre opaque et de la sorte n'existant que pour son mécanisme (régulateur).

Les deux menant, dans un mouvement perpétuel, « une action conjuguée». Mais cela selon ceci que les deux pôles réunis obtiennent leur activation par. l'intervention de la langue: à savoir que cette dernière ( «long ruban de feutre rouge, mais qui pourrait s'avérer au toucher être une sorte de langue" " [p. 79]), fait le lien entre les deux blocs. Et que ce lieu, plus qu'une intervention, peut aussi s'afficher en tant que résultat. II y aurait dès lors à penser que dans l'explicite de ce circuit la convocation des deux pôles ne servirait qu'à faire fonctionner la langue. Mais, ce qui reste à voir : à quel prix?

\section{b) Correction: Faire son temps}

“La fin d'un tel circuit (et son recommencement) est à chaque fois marquée par le déplacement d'un cran du rouage dont l'axe est relié à une tige de fer, formant potence au-dessus du passage de la langue " (p. 80-81). 
Car l'allégorie assimilant le rituel de la pendaison au passage de la langue risque de mener loin le sacrifice. Le risque ici se montre comme tel : faire fonctionner de pair les deux pôles exige de la part de la langue produite un mécanisme correctionnel. II n'y a d'ailleurs qu'à prendre en compte la légende engendrée par l'effet du vent sur le ruban pour attester ce fait : en l'occurrence, il s'agit de violonistes subjugueurs qui, de par leur musique, ont un effet tel sur les enfants que, happés par la langue, ces derniers finissent entre les dents du rouage.

D'ailleurs, l'extension à donner à cette légende prend le pas sur les actuels habitants du village. Ceux-ci, en effet, portent au bras une montre qui fonctionne comme un violon, dont chacun joue et se subjugue en soufflant sur le ruban. Rien d'étonnant donc à ce que d'autres indices viennent étayer le sacrifice, tels:

a) les cubes (blocs) ont un suintement proche de la sueur humaine;

b) «(Quand la pile [de la montre] perd sa charge, nous [villageois] mourons) » (p. 86).

Mécanisme correctionnel donc, le passage de la langue provoque une mise à mort. En ce sens que l'activation conjointe des deux pôles conduit à la formation de la langue ainsi qu'à un rituel sacrificatoire.

Tel semble désormais, en tout équilibre, se délimiter l'enjeu quand, toute récupération admise, est mis en branle le rituel.

\section{Euphorie du discours}

Cette action “conjuguée" ne manque pas de rejaillir ailleurs. Un relais direct au passage d'un récit à un autre s'accomplit entre certaine musique temporelle et la danse devant suivre. Rien d'autre, en effet, à l'avènement de ce nouveau récit, "la Valse des pieds ronds", ne saurait pousser plus loin la combine. Succédant à un mécanisme subjugueur, le rituel avancé sera celui d'un jeu qui intoxique.

\section{a) L'art du dard}

Ici à nouveau, sur la lancée des effets produits par la complicité des deux blocs, s'élabore un champ d'opération qui, des poumons aux orteils, justement fait, en matière de compétition et de récupération, des pieds et des mains. Les extrêmes encore se trouvent ouvertement convoités.

S'agissant d'un jeu, semble se fixer la mise en fonction non compétitive de concepts déjà rencontrés au cours de l'étape antécédente. A savoir que deux camps fort nettement divisés dans des récits antérieurs produisent à la fois leur rencontre et leur fusion. Les éléments devant étayer cette fusion se liraient comme suit :

1) Une «fléchette" composée de bois, de plumes et d'une aiguille, est lancée sans qu'il y ait au préalable de cible; 
2) la "fléchette", une fois piquée sur une matière quelconque, produit, par un mécanisme propre à sa fabrication, sa cible: "Lorsque le projectile frappe son but, la pointe de l'aiguille traverse ce capuchon, retire le cran d'arrêt d'un système de baleines qui déploie la cible automatique, à la manière d'un parapluie» (p. 90).

Cela supposant que la double opération inscrite à même la fléchette (se piquer et produire la cible), dessert aussi bien un jeu de dard qu'un jeu d'art.

Lequel s'esquisse un peu mieux à l'idée que l'action conjuguée de la fléchette et de la cible, au cours d'un même jeu, ravive une distinction établie entre pratique et théorie. Car, si comme cela tend à s'organiser, le sujet (fléchette) produit son objet (cible), il y a toutes chances de croire qu'on assiste à la transgression du jeu de dard en un jeu d'art. Où de surcroît, la pratique produisant sa théorie, l'action conjuguée assure sans compétition une réussite permanente.

\section{b) Une intoxication}

Si, plus haut, les effets entendus dès la rencontre entre ces deux pôles scripturels se traduisaient en un pur et simple affrontement, instaurant d'office un déséquilibre dans quelque pernicieux partage de pouvoir, il y a à craindre que leur fusion mène à des effets d'un autre ordre. Comme telle, la rencontre idéale des deux pôles produit une euphorie sans précédent. Elle se répercutera d'ailleurs, à l'ouverture de la cible-parapluie, sur la personne même du "lanceur". "Le lanceur tombe sur le sol, dans une position de gratitude, les yeux éclatants, la tête renversée. Sa propre voix le secoue et trouble ses compagnons" (p. 91).

Soit un état de transes légitimé par cette euphorie du texte qui, se démarquant du pur et simple affrontement, tombe dans l'intoxication la plus élémentaire :

IIs [les joueurs] se placent au pied d'un escalier de bois et ils lancent la fléchette de sorte qu'elle se fiche dans la contre-marche, qu'elle éclate, puis se referme, en ressorte, tombe en ligne droite sur une marche plus basse, s'ouvre à nouveau comme un pétard d'artifice, rebondisse dans les airs puis se replante dans la marche suivante, et ainsi de suite jusqu'au bas de l'escalier où l'attend, en sueur et en transe, le lanceur, les mains tendues, souhaitant que la fléchette se loge dans l'une de ses paumes offertes pour son ultime éclatement. $(91-92)$

Le sacrifice est de taille. Ici les paumes paument. Inciter le texte à unir deux extrêmes comporte des risques qui imposent à la fois l'intoxication et l'aliénation. L'état de transes, dans son témoignage envers l'euphorie du discours, ne sert qu'à démontrer qu'encore une fois le texte piège (par intoxication) et oblige à la danse, c'est-à-dire à s'y promener sur le bout des pieds. 


\section{L'emboîtement du texte}

Encore que la présence d'un escalier ait identifié le danger, n'y descend pas qui veut. Là réside tout l'art du jeu de dard. Mais qui peut! Si ce n'est dans l'art même celui qui y monte et qui s'y glisse : le rat.

Ainsi donc, pour un nouveau récit, "l'Hôtel ", se redisposent, pour ne pas dire se redistribuent, les éléments servant à alimenter le fonctionnement d'une mécanique dont le rôle est, encore et pour la suite, de rassembler deux pôles.

\section{a) Mise en boîtes}

Les instances mises en place cette fois promettent plus d'agressivité. Partant de "l'Hôtel», se partagent d'abord en toute quiétude des lieux propres à des antagonistes de longue date. Ces lieux sont: un escalier avec glissoire de verre et des chambres avec ascenseurs. Ces antagonistes: des rats qui, de par leur fonction même, permettent l'extension rats d'hôtel (leur rôle n'est-il pas de dévaliser l'hôtel de ses clients), ainsi que des locataires.

C'est dire qu'il y aurait, d'une certaine manière, emmurés dans un même lieu, des éléments faits pour se donner la chasse, lesquels redistribueraient d'emblée les pôles déjà inventoriés à l'apparition des deux boîtes. Tels :

1) Boite transparente: Ce double escalier à glissoire de verre où, d'une part, le verre permet la transparence et où, d'autre part, la danse effectuée par les deux rats peut être perçue de tous pendant toute la journée, ne masquant donc pas là quelque obscur rouage de leur fonctionnement;

2) Boîte opaque: Ces chambres avec ascenseur à contrôle intérieur. Loin ici de s'exposer, et moins encore de se donner en spectacle, ces chambres sont livrées en tant que boîtes suspendues, hermétiques, et donc à l'accès impossible pour les rats. Hors de portée, elles ne trahissent aucun mécanisme extérieur. Telle se lirait en première instarice leur opacité.

Ce qui permettrait, dans le relevé, l'admission de deux boîtes en une seule : l'hôtel.

\section{b) L'hôtel du sacrifice}

Pour ces antagonistes, leur présence réciproque en un même lieu offre l'occasion d'un rituel. Comme toujours l'esprit est au sacrifice. La danse des rats risque, en tout temps, de dégénérer en massacre. Tel d'ailleurs semble être, depuis le début, le prix à payer pour cette téméraire cohabitation. Tantôt de jour, tantôt de nuit, l'autorité change de maîtres. Rats et locataires insinuent le partage, et, d'un même mouvement, s'en voient démunis : “Car ce soir, je le sens, nul ne pourra dormir : ce sera un 
beau spectacle, sanglant et criard, comme le propriétaire les aime" (p. 99100). Puisqu'un troisième angle vient se greffer à la dualité, imposant de par sa position de «propriétaire " la sentence inéluctable à cette cohabitation. Là comme toujours c'en était le risque. Et encore, peut-être plus là qu'ailleurs, puisque l'hôtel serait celui du sacrifice.

Voilà qui peut justifier, passé l'intervention de la langue et d'un discours, une possible activation du narrateur.

\section{Les contraintes du style}

Pareil fonctionnement en cours d'étape ne semble pas près de s'épuiser. Ainsi, sur un autre terrain, en guise d'extension à "l'Hôtel ", le récit suivant "le Manoir de la Taupinière" permet certaines ramifications tant au mécanisme de récupération qu'à celui de correction. Les boîtes, entretenues déjà selon des propriétés de transparence et d'opacité, acceptent une métamorphose en "tiroirs à double fond".

\section{a) En blanc}

Au nombre de deux, et maintenus en parallèle, ces tiroirs s'accordent à perpétuer le fonctionnement de base. Il y aurait de la sorte à envisager que de part et d'autre les pôles sont à nouveau convoqués selon une sommaire description des dits tiroirs :

1) A ranger du côté de la transparence celui qui, inspecté le premier, s'offre comme vide;

2) puis du côté de l'opacité, tel autre contenant un certaìn livre noir.

Comme quoi, en respectant les pôles, le vide fait pencher du côté de la transparence, pendant que le noir, lui, relaie l'opacité. Quant au livre, témoignage d'un “journal personnel", il semble le lieu d'explorations sur le contenu d'un troisième tiroir. Ce qui, dans la mise en parallèle, mène loin l'idée d'un secret. Car il y aurait désormais, selon l'activation de cette mise en parallèle de deux tiroirs nettement distincts, à souligner l'effet disert de quelque nouveau résultat. $A$ savoir: l'apparition d'un troisième secret d'inspection impraticable, à contenu difficile à identifier. Bref le lieu d'une oubliette.

Mais ce lieu a un lien. Lequel tient bon à l'activation des deux tiroirs à double fond. En relation de cause à effet, ce lieu serait maintenu en ce que les spéculations sur le troisième s'enracinent au fonctionnément des deux premiers, à leur action conjuguée. A savoir: l'action combinée du premier et du second tiroir en produit un troisième, comme l'action combinée de l'opacité et de la transparence produisent l'insaisissable, c'est-à-dire un style. Soit, ici, l'aboutissement de toute investigation opérée dans le «journal personnel»: «A la seule pensée de compléter la liste des objets contondants par ce que tout homme de qualité aurait la tentation d'ajouter : ce qu'il y a dans le tiroir secret, c'est un style ${ }^{4} \ldots »$ (p. 111). 
De même que, seconde hypothèse à l'identification du contenu de ce "tiroir secret", se pose en complément de ce style l'idée d'un liquide blanc, sorte d'envers d'encre: «... le bruit du tiroir en mouvement me fait plutôt penser à celui d'un liquide disons enfermé sous vide dans un ballon de verre. [...] un liquide un peu pâteux, d'une blancheur impeccable, très proche du lait au goût» (p. 111). En raison de quoi, le cumul légitimé de ces éléments s'applique à respecter toute blancheur y engageant le caractère insaisissable, voire même secret de tout style.

\section{b) Les travaux forcés}

Le résultat de ces fouilles, de tiroir en tiroir, ne laisse pas indifférent le processus. Ici la découverte reste un prix à payer. Tâter le style jusqu'à l'activer oblige à des sacrifices. Celui qui s'engage à spéculer sur le contenu de ce tiroir secret risque, pour lui-même, la prison à perpétuité. C'està-dire que, voulant sonder cette "oubliette", il s'y verrait relégué. "Les nuits où il n'a pas eu le courage [...] de faire jouer le tiroir, le malade s'est senti comme observé durant son sommeil. [...] plutôt "appelé " par une présence mystérieuse, recelée par le meuble. II a cru, durant quelques pages, à un objet envoûté... » (p. 110) [...] "Peut-être en est-il de même pour ce bureau qui, à en juger par nos affinités, collectionnerait ses propriétaires"(p. 113). Toutes marques dans le texte conduisent à cette pensée: il s'agit bien d'une condamnation. Condamnation à manipuler cette "Oubliette" (tiroir secret), selon cette conséquence que le fouiller c'est en remonter le mécanisme: condamnation à subir cette oubliette selon cet effet en retour que celui qui manipule se trouve sacrifié.

La quête du style donc, et plus précisément sa découverte en combinaison à tiroirs, d'une part, rattachée à son lieu de formation, relègue aux "oubliettes", et d'autre part, formée par sa démarche, condamne aux travaux forcés.

\section{5) À livres... ouvert et fermé}

Avec l'accès à un autre récit, “les Portiers", se déploient de nouveaux raffinements du système. Fortement engagée dans un amas de contraintes, une nouvelle machine mécanise le lieu même où se pose en rituel toute lecture. Travaux ici encore (et pour toujours) de récupération et de correction, les portes, de par leur action conjuguée, capturent par un effet d'emboîtement l'entrée et la sortie du texte.

\section{a) Les portes du pénitencier}

Certaine disposition dans l'amalgame d'éléments sans cesse dédoublés renvoie à la mise en place d'un système complet.

1) Les portes : Données en tant que “porte double à battants ", elles ont d'abord pour fonction d'accomplir un tour complet, et ensuite 
de s'emboîter («à leur rencontre les feuilles de gauche couvrant partiellement celles de droite [puis l'inverse]").

Cela ayant pour but d'empêcher certaine "pénétration par effraction". Le dispositif devant a priori servir de moyen préventif, il sera surprenant de constater à quel point il outrepasse ses droits. Car, loin de simplement prévenir, ce que cette "porte double" propose c'est ni plus ni moins que l'hermétisme: «Pareil hermétisme en ce qui a trait à cette porte est un signe parmi tant d'autres $d u$ peu de cas que les villageois font du verbe entrer et du verbe sortir... " (p. 120).

2) Les portiers: Placés l'un devant, l'autre derrière, ils sont à la fois les agents et les gardiens de ce système. Se ressemblant bien que s'ignorant, ils entretiennent de par la relative analogie de leurs opérations, la plus parfaite confusion entre les termes: entrée et sortie. C'est que, garants de la totalité du système, ils doivent proposer une lecture qui, par emboîtement de feuilles, interminablement recoupe deux livres. Loin d'inviter à une ouverture, ou encore à une fermeture d'un immeuble, c'est à une ouverture-fermeture de livres que convient simultanément les deux portiers.

La récupération s'offre donc avec netteté. Les pôles, par effets de dédoublement, incitent à la confusion : ouverture/fermeture, entrée/sortie (porte double et portier double, l'un devant, l'autre derrière, deux livres...). Mais cette confusion, dans pareil système, suscite, faut-il le répéter, la complétion. Peu de prise en effet s'est laissée en vue d'une reprise des pôles par affrontement. Les feuilles, amovibles, s'emboîtent l'une dans l'autre et se complètent, à perpétuité.

\section{b) À perpétuité!}

Mais à ce titre justement (“les Portiers»), il se produit outrepassement de droits. Quand la fonction d'alors se pose en guise de système clos, à mécanisme suffisant, vient ensuite à l'idée que pareil hermétisme confine à une insinueuse condamnation. Être portier est une chose, mais être portier d'un immeuble inexistant se trouve en être une aùtre. En ce sens que la fonction ici mise en cause préconise une démarche de l'infranchissable. Ces portiers, de la sorte, ne sauraient être autre chose que des gardiens. Gardiens de livres, surveillant les entrées et les sorties, garants de toute lecture, cautionnant avec la mise en marche de ce double mécanisme (ouverture/fermeture) une sorte d'emprisonnement.

Il y aurait là une relation qui de cause, exhibée à l'endroit de certain mécanisme reléguant aux oubliettes, passe indubitablement aux effets. Le livre ici jouerait d'ouverture-fermeture du style, d'extension à son mode de fonctionnement, avec pour effets perpétuels, l'hermétisme, l'infranchissable, l'emprisonnement. Voilà qui déjà pressent quelque appétit carcéral du texte. 


\section{La "Mangeuse de visions"}

Une suite, en ce sens que le système toujours plus clos draine toujours plus la capture, peut être donnée. En effet, l'infranchissable du texte convoque pour sa lecture un autre mécanisme dont la mise au point plutôt que concerner les feuilles s'adresse à l'œil. Encore ici, il s'agirait d'une extension à ce qu'on pourrait nommer une manière de procès textuel. Lequel déjà se reconnaît dans de multiples étapes procédurières, tels les innombrables éléments récupérateurs, ainsi que les directives correctionnelles y étant dévolues.

\section{a) À l'œil!}

Cette ultime étape avant l'apparition du « robot " avive ce qui déjà est effectif. "Le Vaisseau" (avant-dernier récit) contient tous les éléments nécessaires à la reconnaissance d'un nouveau mécanisme favorisant la collaboration des pôles. Ainsi y rencontre-t-on des éléments déterminants :

a) le "vaisseau» est un instrument d'optique à la fois interne et externe:

b) selon sa structure, on réalise que la lentille d'arrivée de cet instrument d'optique est la même que la lentille de départ;

c) cette "sphère de vision" métaphorisée en "araignée de métal et de verre " commande des opérations pour le moins hermétiques: “... l'œil fixé à l'oculaire, ils observent un autre œil en train, quant à lui, d'en fixer un autre, et ceci, presque à l'infini. II peut arriver que l'œil observé soit celui-là même de l'observateur... (p. 127);

d) “Pour bien comprendre la situation stratégique de la salle de contrôle dans l'observatoire, il faut savoir que l'immeuble cubique est tranché en deux parties égales par un mur immense..." (p. 130). Cela résultant en deux ailes : aile $A$ pour les chambres; aile $B$ pour la salle de contrôle.

Aucun doute, donc: nous sommes en terrain déjà balisé.

\section{b) OEil pour œil}

Un tel état de texte doit mener vers des effets déjà reconnus, puisque c'est à répétition que s'engage, depuis l'amorce de ces multiples étapes devant mener au troisième robot, le mécanisme correctionnel. Répété mais non analogue. Les étapes franchies arborent sans cesse une nouvelle commande. Ainsi se pose-t-il pour ce récit précis des aménagements carcéraux caractérisés.

D'abord il faut savoir que sont mis en relation "observateurs" et "contrôleur"; c'est-à-dire ceux dont le travail consiste à prendre des notes à partir d'observations sur l'œil vu (un vocabulaire technique assure la neutralité de ses commentaires), et celui dont le rôle est de recueillir les données et d'effectuer des corrections: «... c'est lui qui, bénévolement, 
effectuera les corrections nécessaires sur l'instrument d'optique et verra à son entretien [...] Par une vigilance de tous les instants, l'opticien de service remplacera les sujets défaillants... » (p. 132).

A savoir que le contrôleur se trouve en position de pouvoir. Pouvoir de correction. sur la machinerie, mais pouvoir également sur les «observateurs " dont souvent, on peut observer le caractère délateur des notes recueillies. A savoir aussi, toujours selon une optique carcérale du texte, que les chambres où logent les divers observateurs «ont plutôt l'aspect de cellules, leur exiguïté empêche la présence de toute pièce de mobilier hormis le lit et la chaise, sans parler du tube périscopique" (p. 128).

Voilà qui met en lumière une relation d'observateurs à contrôleur qui s'applique à imposer un pouvoir optique en vue de certain assujettissement. En clair, le mode d'utilité du «vaisseau " affiche un mode de correction: “ll y en a qui prétendent que cette araignée monstrueuse et inesthétique [sic] est une "mangeuse de vision", qu'elle ne sert qu'à nourrir un organisme artificiel conçu en marge d'une campagne de promotion lancée par les opticiens dans le seul but de voir proliférer les commandes de verres correcteurs" (p. 133).

Correction de la vue par les verres, ce "vaisseau", ce texte, cette araignée apposée sur sa lecture, se veut “mangeuse de vision». Pareil châtiment, pour qui joue l'observateur, souligne de façon outrancière la préserice permanente d'un complot. Les "verres correcteurs" corrigent, indubitablement, et par le fait même obligent et sacrifient.

Le "Je" se prothèse

A nouveau, quoique pour une dernière fois, vient se dresser en bout de lecture un robot. Identifié comme "le Bernard-I'Ermite (Robot III)", il n'est pas sans marquer l'état robotisé de la troisième étape, ainsi que la position définitive du texte. Dernier d'un groupe de trois, ce robot, en effet, appuie l'idée qu'une tendance à l'envisager comme résultat ultime du texte prévaut.

Afin de bien cerner cette situation, il faut orienter la lecture de manière à ne rien négliger de deux directions élémentaires : l'une figurant à grands traits l'état confirmé de la dernière étape (dans ses recoupements avec la récupération et la correction), l'autre clarifiant par une mise en relations l'importance stratégique de ce troisième robot par rapport aux deux autres.

\section{a) Libération carcérale}

Dans le vif donc, à compter de la «cage" (p. 137), s'interposent certaines données devant permettre d'engager la participation de deux pôles. S'agissant d'abord d'une prothèse, sa disposition étonne tant sa qualification que dans son adresse au corps. Ainsi, faut-il savoir que déjà en son vocable, la “cage libératrice» arbore des pôles. Cage d'une part, et 
libératrice de l'autre, elle unit des inconciliables, et ainsi permet l'un par l'autre une récupération immédiate. De plus, en tant que telle cette "cage» n'est pas, bien que figurant dans le "catalogue", une prothèse. Obligeant en effet l'expérimentateur à faire corps avec elle pour qu'elle puisse fonctionner, elle permet le fondu de deux éléments fort distincts: l'expérimentateur d'une part, et la cage de l'autre. Puis, une fois cette opération effectuée, elle rétablit le fonctionnement des pôles, selon que désormais sont accouplés dans un même processus le corps de l'encagé et le moteur: "... une autre personne, de l'extérieur, sangle le cobaye fermement, puis barre la portière. Le verrou une fois en place enclenchait les engrenages du moteur situé aux pieds de la cage dont les barreaux suivaient abruptement mais fidèlement les configurations anatomiques de l'encagé " (p. 138).

Relation immédiate, moteur et encagé supportent un fonctionnement qui, de cause à effet, garantit la plus totale satisfaction. Mais cela ne va pas sans accroc. Ça s'est vu en de nombreuses circonstances déjà ; les unions illicites poursuivent des fins tragiques. L'ambiance carcérale des derniers récits en témoigne déjà beaucoup. Nulle surprise donc à ce que la relation cage/encagé déjà tende à réinvestir une quelconque méforme correctionnelle: «... l'apparence de la cage se modifiait au gré de son prisonnier...» (p. 138).

Pour sûr les moyens de libération recèlent des fins mal avouées. Aussi le rapport entre les pôles subira-t-il d'infimes déplacements. De cage à encagé d'abord, puis d'encagé à moteur et enfin de cage à prisonnier, se démarque une quatrième instance où s'effectue la disparition provisoire du prisonnier: elle a lieu à l'occasion de la manifestation de l'automate identifié à l'effet d'un "Je», et ayant pour rôle d'entretenir et de faire fonctionner le moteur. II en résulte une relation de couveur à couvé; l'automate ("Je») couvant le moteur reposant sur un socle ressemblant à un coquetier.

\section{b) Dans le camp de concentration}

Le glissement déjà relevé de l'encagé au prisonnier annonce la procédure à suivre. Certaine fonction de la cage piège, déjà, par inversion. Trois étapes, comprises dans les inversions développées à même le chapitre, concrétisent l'apogée du mécanisme correctionnel :

1) Cataloguée en tant que "démultiplicateur", la "cage libératrice" se révèle être un «compresseur»: l'être s'y comprise «petit carré aplati et luisant, incrusté à même la carapace du moteur" (p. 138).

2) Entreprise de Libération, la cage, par abus de concentration, confine au camp: «Cette prothèse, à bien y réfléchir, en était une en sens inverse : elle privait plutôt que gratifier, et par là, elle ouvrait à notre petite collectivité la voie de l'ascèse, de la solidifica- 
tion, du retour au noyau primitif " (p. 139); proposant par là une manière de «migration par le dedans". Dans une pratique à fort accent commercial, le catalogue d'abord, la cage ensuite manifestent outrageusement des effets de fausse représentation. Laquelle représentation en prend d'ailleurs pour son référent quand le moteur s'active.

3) Perte référentielle, les hommes rapetissent et sombrent dans une noirceur de plus en plus hermétique. Peu à peu envahies par la cage libératrice, les autres prothèses disparaissent du catalogue.

4) Seuls l'automate et la cage libératrice demeurent, se partageant les feuillets du catalogue. Mais il s'agit ici et pour longtemps, d'un automate "Je", publiant à chaque année ses "feuillets", automatisé dans l'écriture, robotisé par le texte complice de son moteur, machinant un ultime mais inéluctable complot.

\section{c) Une prison textuelle}

En guise d'aboutissement aux rituels enclenchés au cours des six "récits" précédents, ce troisième robot dirige la lecture. La mise en procès du texte est aussi une mise en procès par le texte. La lecture, prise en souricière, trébuche sous le coup d'une intense machination. Elle devient, sous l'influence de ce guide automate ( Je»), petit à petit, machinale. Et, bien que conduisant au bonheur ceux qui expérimentent la "cage libératrice", la lecture n'en demeure pas moins un danger, sorte de bombe expansive pouvant en tout temps faire sortir les villageois de leur réduction, et les propager tout azimut.

Encore une fois c'est le risque à tout rituel euphorique. Et ce dernier robot en souligne encore plus le caractère. Le monde concentré et compressé de l'écriture comporte des dangers plus grands que la lecture ne le suppose. Partout l'éclatement veille. Malheur à qui s'en est imprégné, car il ne saura jamais quand se produira sa propagation dans le texte.

Quelques considérations encore poursuivent un semblant de portrait pour ce troisième et ultime robot. Elles touchent certains recoupements entretenus avec le deuxième, marquant par là une suite stratégique de la ligne à suivre.

Partant en effet de ce que le second robot rétablissait, selon un précaire équilibre, un affrontement consommé entre référence ( Je») et moteur, compte tenu que ce dit moteur, était alors un extracteur de Jus/Je; il appert que désormais une étroite collaboration entre les éléments confrontés s'est établie. En effet, ici moteur et “Je" mettent en marche et en marché la cage libératrice. A savoir ici que la "cage libératrice" poursuit le travail de compression déjà amorcé sur le «Je" lors du huitième récit, mais sur d'autres éléments cette fois, et avec la collaboration de ce même "Je". Avec, en guise de complément certaine progression dans l'assujettissement du texte qui, noyé toujours plus de noirceur, penche définitivement du côté de l'opacité. 
Sans plus de référence aucune, ce “ Je » aura, tout au cours du texte, franchi maintes étapes devant le textualiser. Parti d'un robot, le premier., récupérant son message, puis passant à un second affairé à lui extraire toute référence, il aboutit, complètement automatisé, dans un catalogue tout aussi machinique que l'œuvre elle-même.

Toujours s'obscurcissant, attíré et dirigé par la noircẹur, ce «Je», ultime, par une sorte de penchant vers les mots (noirs et hermétiques), finit par rejoindre le texte. Bien sûr, il fait figure de prothèse mais d'une prothèse faisant corps avec le catalogue.

\section{Vers l'opacité}

Dans l'entier parcours de l'œuvre un combat se mène. Se départageant quelques dimensions du texte, référence et fiction s'affrontent au gré de l'écriture. C'est à qui motivera la plus large part d'influence. Trois robots, on le sait, rendent un compte rigoureux de cette machination. Les modes, d'ailleurs, en appellent la diversité. Tantôt pour le "Robot I", c'est l'opposition qui prévaut; tantôt, avec «Robot II », un déséquilibre s'instaure; tantôt, avec "Robot III", domine une mécanique correctionnelle. Soit un parcours en étapes irrégulières qui, mettant en scène des pôles, produisent une pernicieuse stratégie. Voilà qui, en tous lieux, jaillira sur la conduite de lecture.

Il y a, comme à l'abord, un texte en contradiction. Des systèmes, des boites, des concepts même s'opposent, voire à grands coups d'utile et d'inutile, se livrent un étrange combat. Répertoriés, de manière à figurer au tout premier plan, ils ont pour mérite d'exhiber les instances de la composition. Tout travail en effet, du moment où il se destine à l'écriture, ne peut s'empêcher de produire ces deux dimensions. C'est un inévitable. Comment, cependant, il s'applique à les produire, voilà qui marque à l'intérêt un certain étranglement.

Ici la composition vit en célibataire. C'est-à-dire que, donnée comme manufacture, elle s'élabore autour d'un mécanisme qui fait que les pôles (référence/fiction, théorie/pratique), s'attirant et se repoussant, marquent une autosuffisance. Le fonctionnement du texte (qui est fonctionnement de tout texte) produit sa propre démarche. Ou plutôt : sa démarche serait de produire son propre fonctionnement.

Ce qui, somme toute, ne semble pas tout à fait nouveau. L'idée, par ailleurs, admise à cette machination, que le texte sacrifiant un rituel s'instaure, fait passer du côté de l'approbation l'égard à une telle démarche. Célibataire dans sa production, dont l'autosuffisance vise, plutôt qu'une reproduction, un travail en réduction (voir la "cage libératrice"), ce texte permet de rendre compte des effets dévastateurs des concepts agissant dans l'écriture. Les risques, avec les résultats qu'on connaît, ont été 
courus. Passant d'une opposition de systèmes à une inversion dans le pouvoir, le texte aboutit à un monde concentrationnaire, marqué par l'ascèse et l'hermétisme. La démarche, toujours plus, confine au noir, rongeant par là même l'essentiel de la dimension transparente. En bref, le travail de fiction anéantit celui de la référence. Ce qui, en manière de conséquence, n'autorise nulle sortie du texte. Donné comme manufacture, c'est autant le mode de production que la production elle-même (machines) qui légitiment cette dangereuse démarche.

Stratégique en effets, cette scription, à vouloir exhiber de façon tantôt oppositionnelle, tantôt intersectée, ses propres dimensions (théorie/ pratique, référence/fiction), tend à se donner tous les rouages de la mécanique. Invitant l'écriture à se nourrir de ses propres mécanismes, elle attire l'attention sur un travail de mixité qui, dans ses effets de texte, obligent à certains risques. Ainsi les corrections, ainsi les sacrifices. La référence en prend pour son soûl! Ce qui n'empêche pas pour autant le texte, comme machine, de mourir de désir pour lui-même.

Ghislain Bourque, Université du Québec à Chicoutimi.

1. L.-P. Hébert, la Manufacture de machines, Montréal, Les Éditions Quinze, 1976, $143 \mathrm{p}$.

2. Les Machines célibataires, Venise, Alfieri, 1975; voir l'article de Michel Carcouges, p. 21.

3. Outre le troisième récit, voir aussi le huitième, "l'Extracteur de jus (robot II)", p. 67 et le quinzième, "le Bernard-I'Ermite (Robot III)", p. 135.

4. Souligné dans le texte. 